\title{
I vasi « acustici » ed i bacini della chiesa di S. Giovanni (Shën Jan) a Delvinë (Albania)
}

\section{Suela Xhyheri}

\section{Q OpenEdition}

1 Journals

\section{Édition électronique}

URL : https://journals.openedition.org/cem/13295

DOI : $10.4000 /$ cem.13295

ISSN : 1954-3093

Éditeur

Centre d'études médiévales Saint-Germain d'Auxerre

\section{Référence électronique}

Suela Xhyheri, «I vasi « acustici » ed i bacini della chiesa di S. Giovanni (Shën Jan) a Delvinë

(Albania) », Bulletin du centre d'études médiévales d'Auxerre | BUCEMA [En ligne], 17.2 | 2013, mis en ligne le 13 décembre 2013, consulté le 22 septembre 2022. URL : http://journals.openedition.org/cem/ 13295 ; DOI : https://doi.org/10.4000/cem.13295

\section{Ce document a été généré automatiquement le 22 septembre 2022}

\section{c) (i) (2)}

Creative Commons - Attribution - Pas d'Utilisation Commerciale - Partage dans les Mêmes Conditions 4.0 International - CC BY-NC-SA 4.0

https://creativecommons.org/licenses/by-nc-sa/4.0/ 


\title{
I vasi « acustici » ed i bacini della chiesa di S. Giovanni (Shën Jan) a Delvinë (Albania)
}

\author{
Suela Xhyheri
}

1 L'obiettivo del presente articolo è quello di discutere una serie di vasi « acustici » e di «bacini » provenienti dalla chiesa di San Giovanni a Delvinë (Albania), inseriti nella costruzione e databili tra X-XII secolo. Per quanto riguarda le ceramiche « acustiche » il tema è stato finora trattato solamente da T. Gjoka ${ }^{1}$, ma esclusivamente in quanto amplificatori sonori; senza tener conto del più ampio

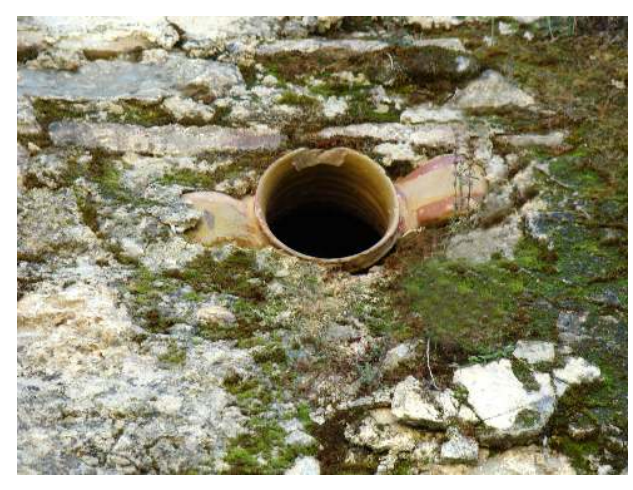
quadro storico-archeologico, e del loro maggiore interesse nel quadro degli studi sul « Bauforschung ».

2 Le rovine della chiesa di S. Giovanni a Delvinë si trovano su una bassa collina, situata nel nord-ovest del territorio dove si estendeva la città antica di Foinike, vicino alla riva del fiume Kalasë. Nel XX secolo nelle vicinanze delle rovine della chiesa fu insediato un nuovo villaggio chiamato Shijan. Gli scavi hanno portato alla luce un ricco materiale archeologico, che permette di ricostruire le varie fasi della costruzione e di avere una idea generale dell'architettura del monumento ${ }^{2}$.

3 La pianta, conservata in buono stato (fig. 1), le tecniche di costruzione delle mura, l'arredo liturgico, la scultura architettonica, l'apparato decorativo, la pittura murale, il pavimento posato con opus sectile ed il materiale archeologico, indicano quattro fasi principali di costruzione. 
Fig. 1 - Planimetria della basilica di San Giovanni (MUÇAJ et alii, « Rezultatet e gërmimeve... », 2004, p. 95, fig. 2)

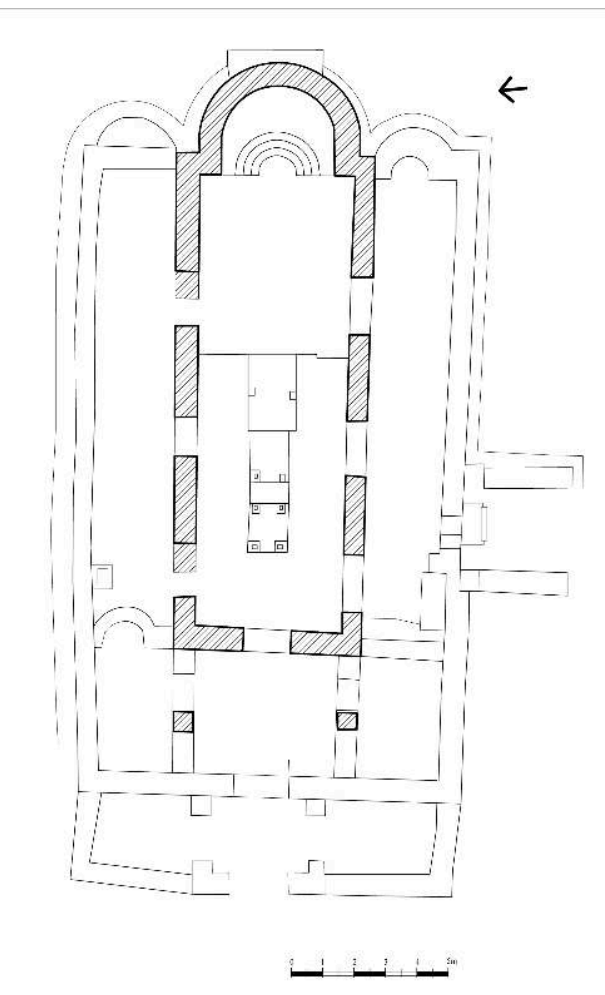

La chiesa a nartece è stata costruita inizialmente, per poi trasformarsi in una basilica a tre navate (seconda fase). La costruzione dell'exonarthex appartiene alla terza fase, mentre la demolizione della chiesa annessa alla parte nord del nartece e la conseguente costruzione della chiesetta segnano la fine dell'attività costruttiva (quarta fase). Gli autori degli scavi hanno definito il periodo per ciascuna delle fasi : prima fase (IX-X sec.), seconda (inizio XI sec.), terza (XIII sec.). Un tesoro di monete trovate nello strato contenente cenere, che si chiude con la moneta di Bartolomeo Gradenigo (1339-1342), indica che la chiesa è stata bruciata durante le incursioni intraprese da Stefan Dushan in questi territori - tra la fine del 1342 e l'inizio del 1343. Dopo la sua distruzione, la basilica è stata adattata in una chiesa a navata centrale ed utilizzata per un breve periodo nella seconda metà del XIV sec.

5 San Giovanni a Delvinë è la prima chiesa medievale in Albania ad avere ben conservato le installazioni liturgiche: il syntronon, il ciborium, il parapetto del santuario e l'ambone, collocato in asse e collegato ad una solea, come da tradizione paleocristiana di Costantinopoli. All'interno della chiesa, specialmente presso le campate laterali, sono state trovate una grande quantità di tombe con la copertura a volta e sarcofago. Gli scavi eseguiti nella basilica di S. Giovanni a Delvinë hanno portato alla luce innumerevoli frammenti di affreschi appartenenti a tre strati diversi ${ }^{3}$.

6 La ceramica rinvenuta è quantitativamente scarsa e composta da: pithos, anfore ${ }^{4}$, bottiglie, ceramica da tavola invetriata e ceramica da cucina. Come materiale da costruzione, a parte i mattoni e le tegole, troviamo le "bottiglie" (fig. 2-5), le anfore (fig. 6) usate a fini acustici ${ }^{5}$, ed i vasi invetriati utilizzati come elementi di decorazione (fig. 7-9). 
Fig. 2 - Bottiglie « acustiche » di Delvinë n 1-5 (S. Xhyheri).
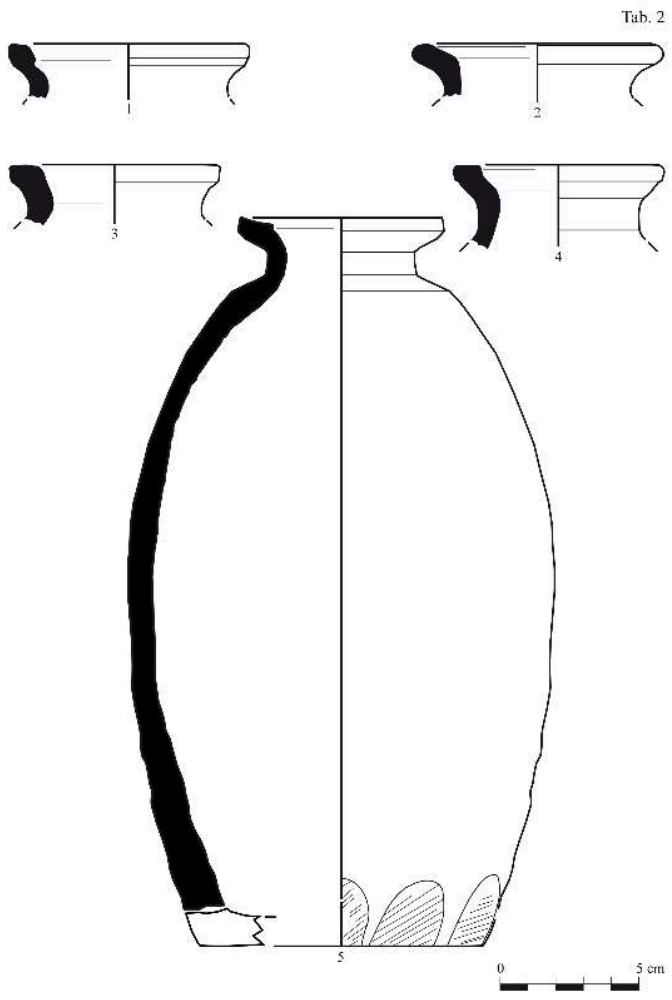

Fig. 3 - Bottiglie « acustiche » di Delvinë n 6-11 (S. Xhyheri).

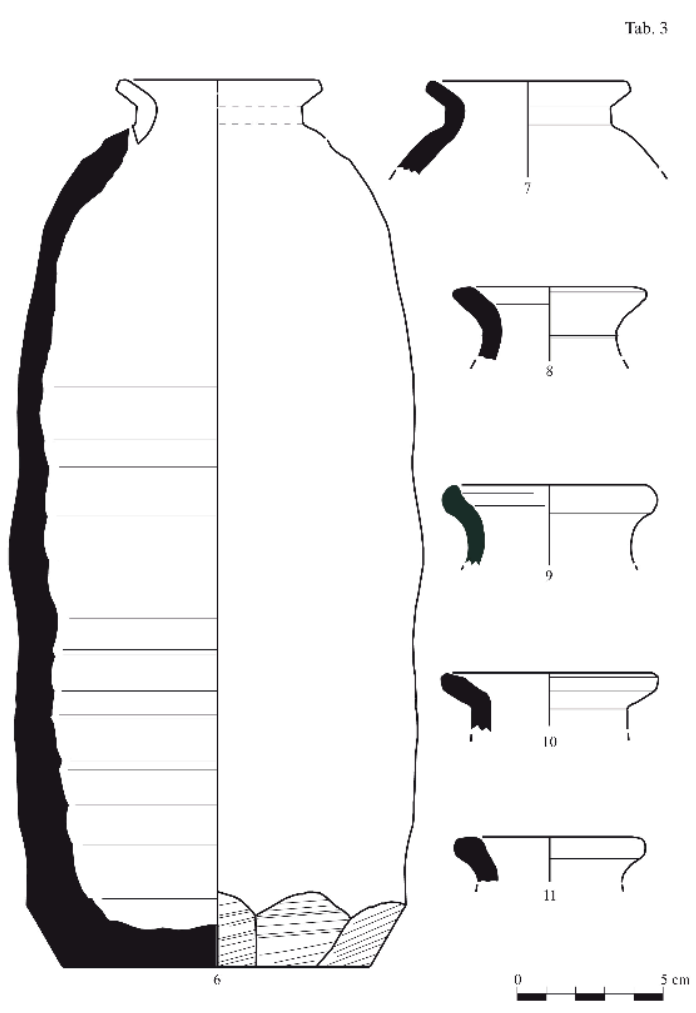


Fig. 4 - Bottiglie « acustiche » di Delvinë n 12-17 (S. Xhyheri).
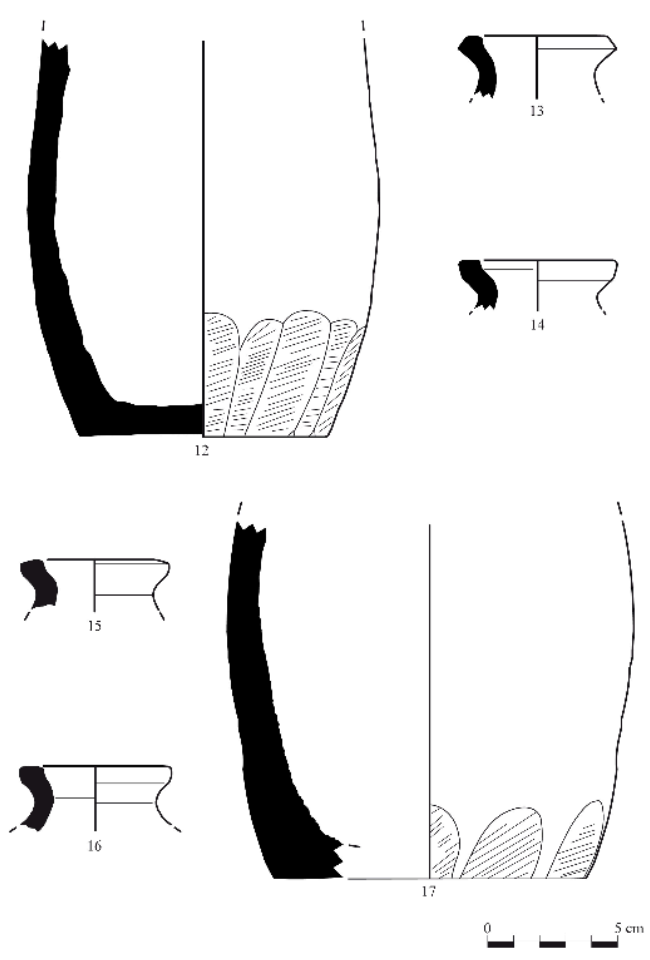

Fig. 5 - Bottiglie « acustiche » di Delvinë n 18-23 (S. Xhyheri).

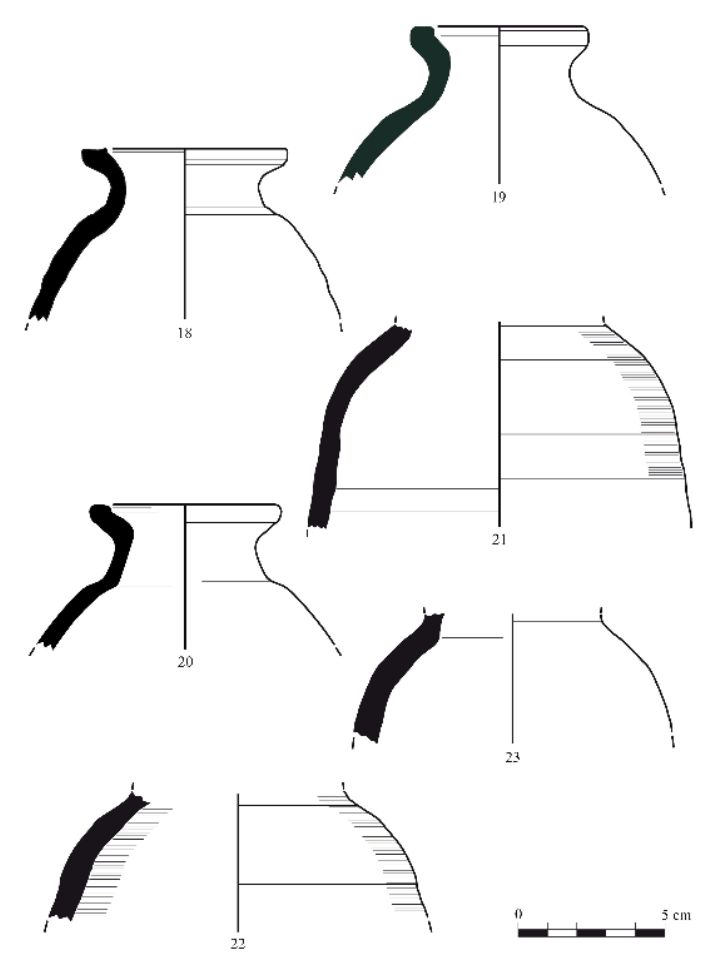


Fig. 6 - Amfora « acustica » di Delvinë $n^{\circ} 24$ (S. Xhyheri).

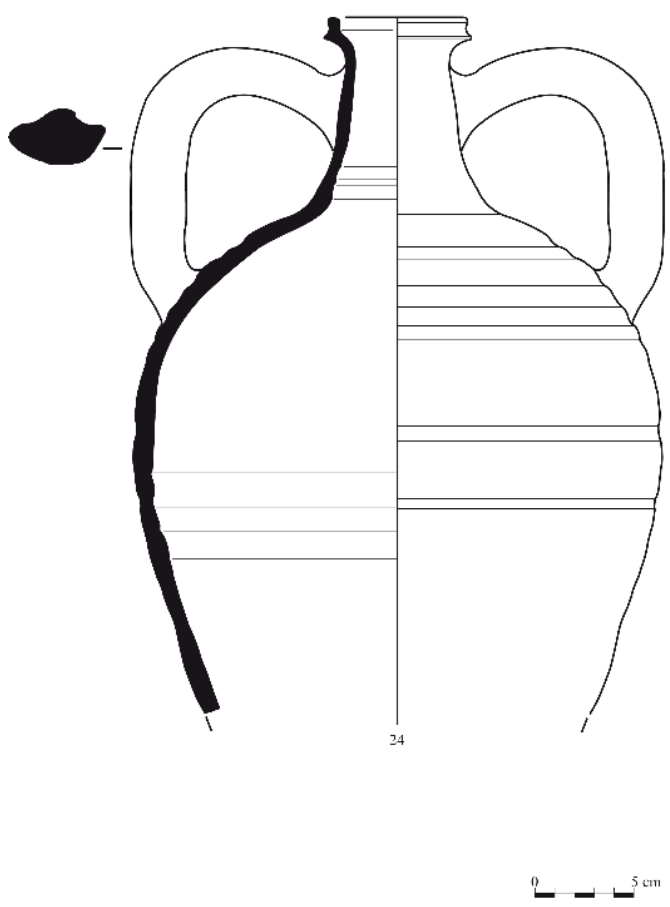

Fig. 7 - Vaso « acustico » in situ nella chiesa di Krye Engjëjve, Voskopojë (Korçë ; S. Xhyheri).

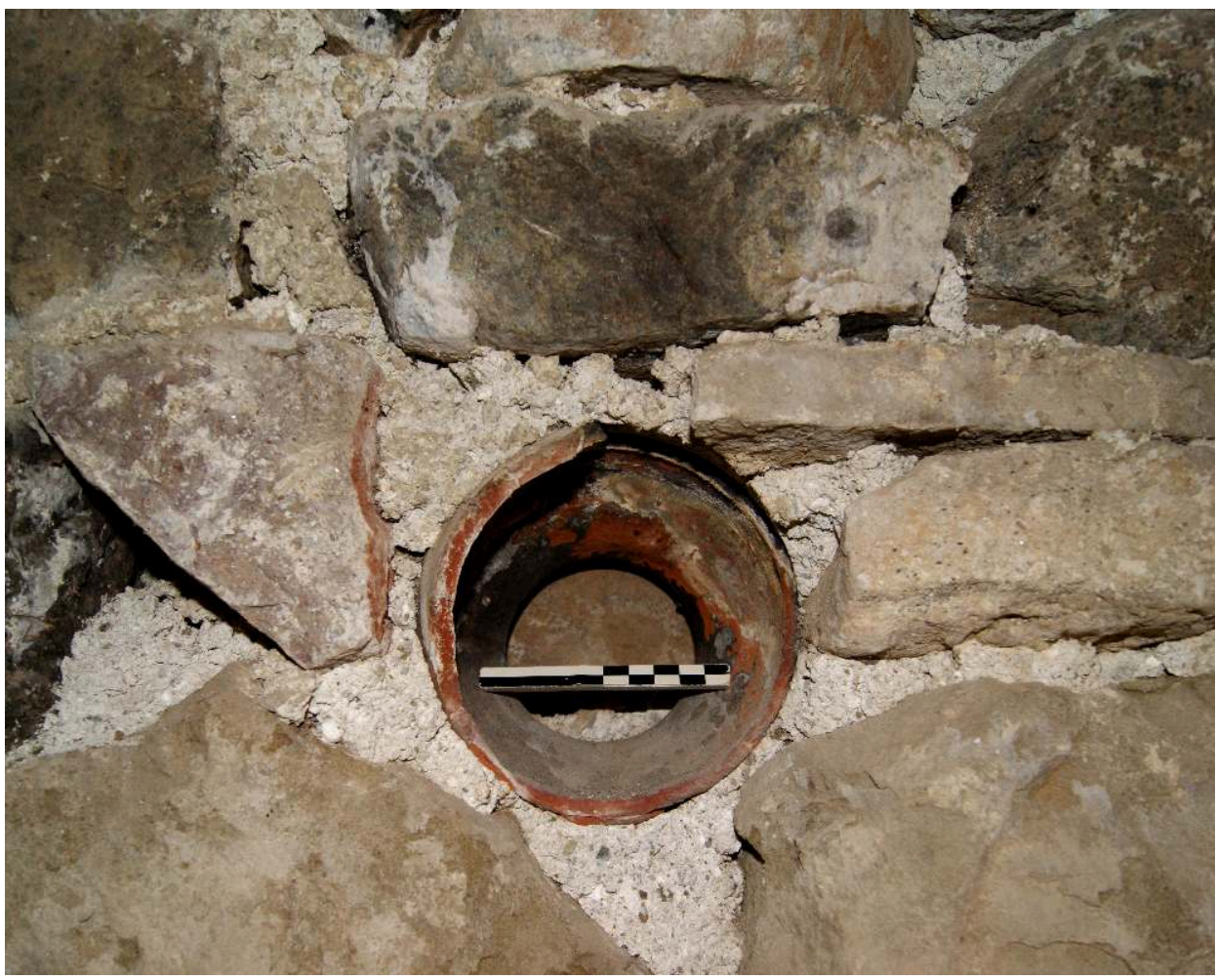


Fig. 8 - Vaso « acustico » in situ nella chiesa di Ndroq, Tiranë (S. Xhyheri).

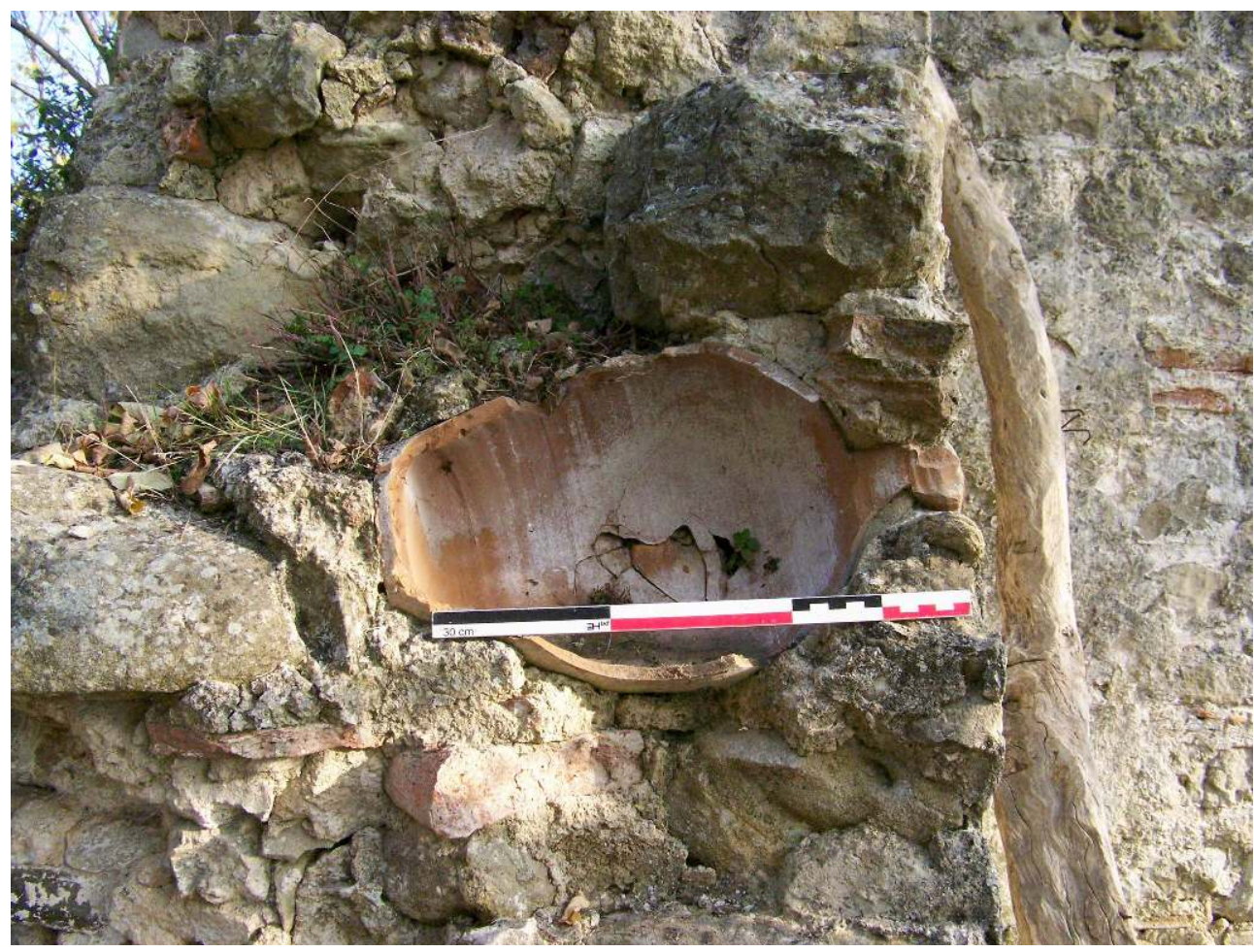

Fig. 9 - Vaso « acustico » in situ nella moschea di Xhermëhallës, Delvinë (S. Xhyheri).

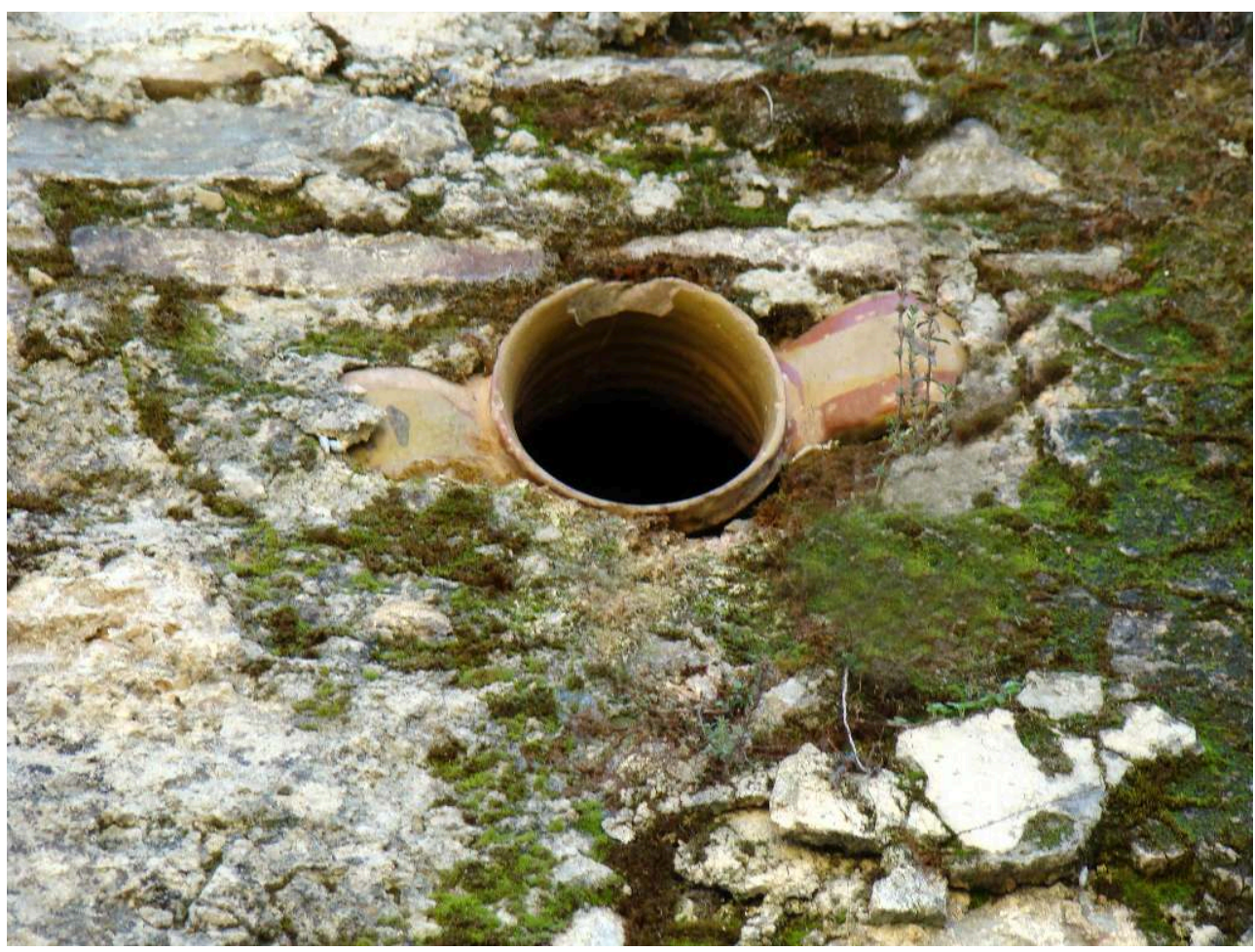

7 Frammenti di bottiglie ed alcune anfore (fig. 6, $\mathrm{n}^{\circ} 24$ ) con addosso attaccata la malta, sono state trovate nelle vicinanze dei muri della navata principale - solo qualche frammento disperso si trova appartato, durante i periodi di ricostruzione o di apertura delle cripte. Le bottiglie hanno la parte dell'orlo pitturato con lo stesso colore dei muri 
della chiesa, ulteriore elemento, oltre alla malta, che rafforza l'ipotesi della loro inserzione murale. Il loro ritrovamento assieme ai pezzi del muro crollato, con macchie di malta e di pittura, indicano che erano messe all'interno del muro, con la parte finale dell'orlo sporgente sulla superficie, ed utilizzate a scopo acustico. Nella chiesa di S. Giovanni a Delvinë, a parte i materiali acustici sopra elencati, di particolare interesse sono alcuni vasi che conservano i segni della malta con i fori sul fondo (fig. $7, \mathrm{n}^{\circ} 25$ ), che erano incastrati nel muro. Questi ultimi non solo indicano la data del loro uso in Albania, ma aiutano a stabilire il limite temporale dell'utilizzo dei vasi a fini acustici nelle chiese. Mettendo a confronto la possibilità di datazione che offre il materiale archeologico a scopo acustico, con la struttura della chiesa, il loro utilizzo risale alla prima fase di costruzione (la chiesa a navata unica).

8 La chiesa a nartece (fig. 1), di forma rettangolare di dimensioni lunghezza $14,35 / 14,50 \mathrm{~m}$ e larghezza $4,75 / 4,95 \mathrm{~m}$, aveva un abside semicircolare e un protiro monumentale nel lato ovest. L'abside nella prima fase aveva il synthronon con una scalinata semicircolare con tre pedate, che conducono a una platea di legno ${ }^{6}$, mentre per le altre installazioni al suo interno - il ciborio, il parapetto del santuario e l'ambone -, allo stato attuale delle conoscenze non si può affermare se facevano parte della chiesa a nartece, oppure se appartengono alla seconda fase di costruzione - la trasformazione della chiesa in basilica ${ }^{7}$.

Due pilastri di granito a sezione rettangolare alti $3,50 \mathrm{~m}$, riutilizzati nel pavimento della seconda fase, dovrebbero appartenere al protiro monumentale dell'entrata ovest. Le altre due entrate disposte al lato nord, conducono una al santuario e l'altra al nartece.

\section{I vasi « acustici »}

Le bottiglie e le anfore utilizzate nella costruzione della chiesa - prima fase : fine IX sec.-inizio X sec. -, sono state trovate frammentate e appartengono a 35 esemplari, dove le bottiglie sono le più numerose (fig. $2, \mathrm{n}^{\circ} 5$; fig. $3, \mathrm{n}^{\circ} 6$ ).

11 La loro forma particolare lasciava inizialmente supporre che si trattava di un caso unico d'utilizzo a scopo acustico. I numerosi esemplari rinvenuti nella chiesa di S. Giovanni a Delvinë, negli scavi di Siena ${ }^{8}$ e Montarrenti ${ }^{9}$ (Italia Centrale), simili ai nostri, indicano invece che si tratta di comuni vasi da tavola, mentre usati a fini acustici li troviamo solo nel nostro caso.

12 Le bottiglie sono state preparate con argilla colorata ${ }^{10}$ con tinte in sfumatura, che va dal giallo chiaro al marrone molto chiaro. I vasi hanno forme simili con le comuni bottiglie di vetro, e gli esemplari restaurati hanno un'altezza che varia da $26-30 \mathrm{~cm}$. Il loro corpo ha una forma prolungata con il fondo piano e massiccio, con un particolare : le pareti laterali fino a $8-10 \mathrm{~cm}$ nella parte bassa non sono circolari, ma filettati, realizzati a taglio con coltello prima della cottura (fig. $4, n^{\circ} 12$ e 17). Le bottiglie hanno il collo corto e l'orlo a imbuto con un diametro esterno 5,9-6,2 cm (fig. 2, nº 1-4 ; fig. 3, $\mathrm{n}^{\circ}$ 7-11; fig. 4, no 13-16; fig. 5, n 18-20). I vasi della chiesa di S. Giovanni a Delvinë, a parte le somiglianze formali con quelle di Siena e Montarrenti, hanno lo stesso fondo con pareti diritte, sporgenti verso l'esterno, filettate e tagliate con coltello verso il fondo ${ }^{11}$.

13 Assieme alle bottiglie sono stati trovati frammenti di 12 anfore diverse, parti di: maniglie, collo, parete, spalla e fondo. Le anfore appartengono a diversi tipi italo- 
bizantini, simili alle anfore del X-XI sec. rinvenute in Reggio Calabria ${ }^{12}$, tipo Otranto $1 \mathrm{e}$ Güsenin $1^{13}$.

14 L'utilizzo della ceramica acustica si riscontra in tutto il Mediterraneo ${ }^{14}$, specialmente nelle chiese del X-XIV sec., mentre nel territorio albanese reperti simili sono presenti nei monumenti a partire dal XVI sec. Esempi conservati in situ si ritrovano nella chiesa di Krye Engjëjve nel villaggio di Voskopojë a Korça (fig. 7), nel villaggio di Ndroq a Tirana (fig. 8) e nelle moschee di Xhermëhallës (fig. 9) e di Gjin Aleksit a Rusanj di Delvinë (fig. 10). L'unico caso del loro utilizzo in periodi antecedenti è testimoniato nelle fonti scritte albanesi ed appartiene alla chiesa di Shën Kollit (S. Nicolo) di Armen ${ }^{15}$.

Fig. 10 - Vaso « acustico » in situ nella moschea di Gjin Aleksit a Rusanj, Delvinë (S. Xhyheri).

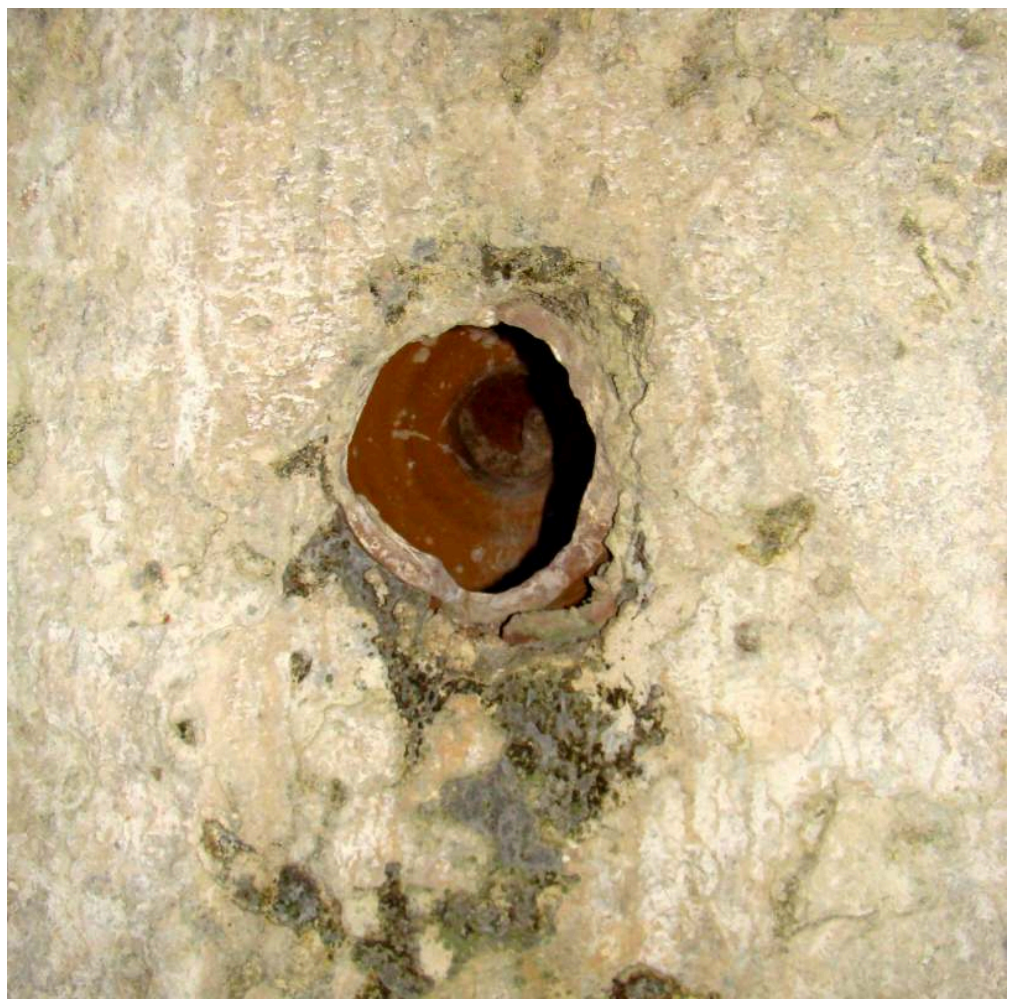

15 Gli scavi nella chiesa di S. Giovanni a Delvinë non solo hanno contribuito ad arrichire la varietà delle tipologie dei vasi utilizzati a fini acustici, ma aiutano a stabilire un limite temporale anteriore rispetto a quello esistente nel territorio albanese. I vasi utilizzati come bacini appartengono alla ceramica invetriata del tipo "polychrome ware " ${ }^{16}$, e sono ornamentati con motivi floreali di colore verde, giallo (fig. $11, \mathrm{n}^{\circ} 25$; fig. $8, \mathrm{n}^{\circ} 26 \mathrm{e}$ 27) e marrone (fig. 13, $n^{\circ} 28$ e 29). 
Fig. 11 - « Bacino » di Delvinë n 25 (S. Xhyheri).
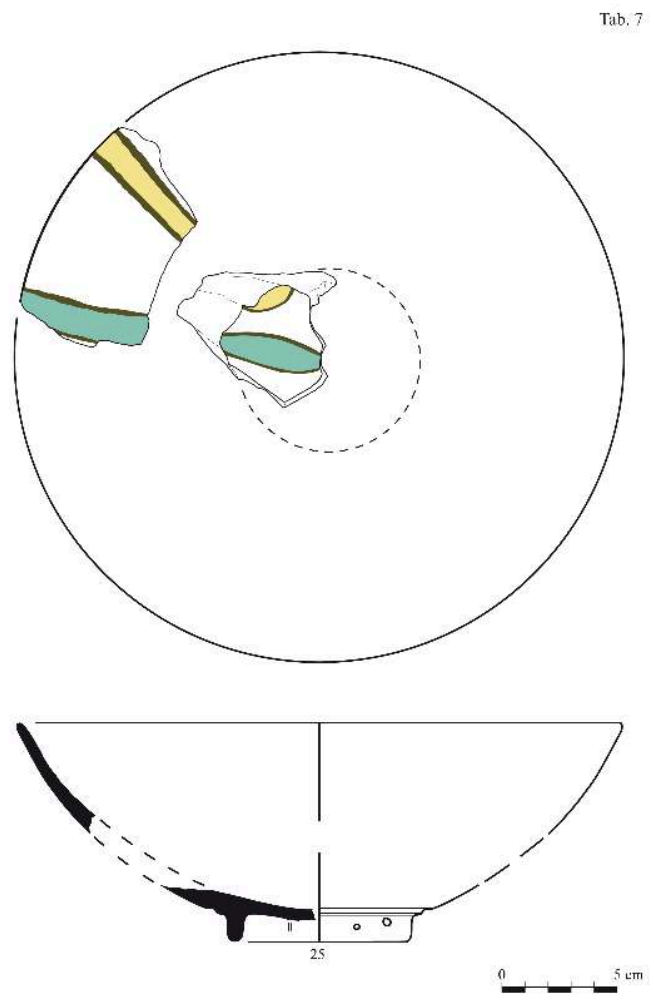

Fig. 12 - « Bacini » di Delvinë n 26-27 (S. Xhyheri).

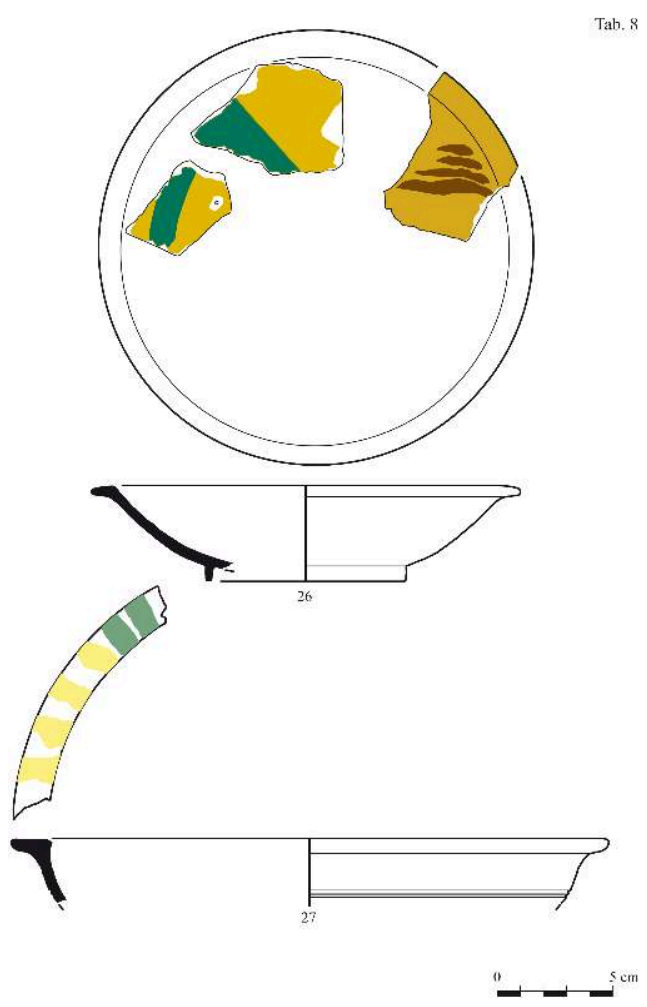


Fig. 13 - « Bacini » di Delvinë n 28-31 (S. Xhyheri).

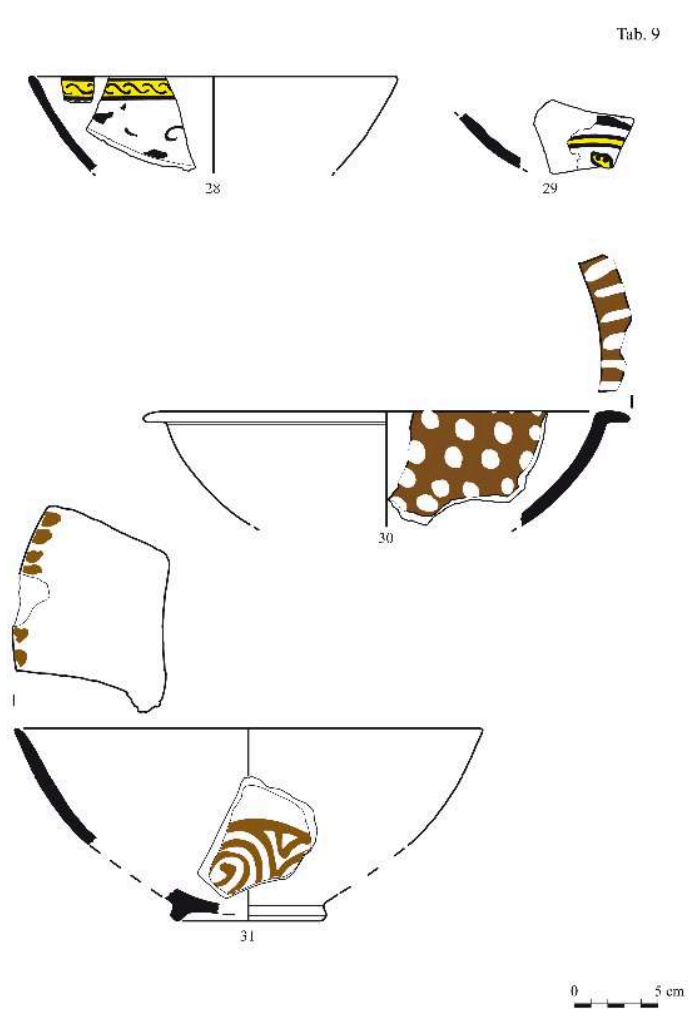

Il primo gruppo dei vasi è comune del periodo compreso tra la fine del X sec. e l'inizio del XI sec., ma si può anche trovare depositato nei strati dell'inizio del X sec. ${ }^{17}$. I vasi appartenenti al secondo gruppo, ornamentati con colore marrone ${ }^{18} \mathrm{e}$ giallo, si possono datare verso la fine del XI sec. ${ }^{19}$. I vasi «polychrome ware » del primo gruppo hanno somiglianze evidenti con quelle invetriate di piombo del IX sec. provenienti dal Medio Oriente ${ }^{20}$. A parte i vasi del X sec., abbiamo un altro gruppo appartenente al tipo «slip painted ware $"{ }^{21}$ con ornamenti a spirale messi in risalto dal contrasto chiaro scuro (fig. 13, $\mathrm{n}^{\circ} 31$ ), nonché un ciotola invetriata color marrone decorata all'interno con puntini bianchi del tipo «dotted slip painted ware » (fig. 13, $\mathrm{n}^{\circ}$ 30). Questo gruppo si può datare tra la fine del XI sec. e l'inizio del XII sec. I bacini del primo gruppo combaciano con la prima fase costruttiva della chiesa di S. Giovanni a Delvinë, rendendo possibile sia la datazione della chiesa a un nartece - fine del IX sec. ed inizio del X sec. -, che quella dei vasi « acustici ». L'altro gruppo dei bacini dovrebbe essere stato utilizzato nelle fasi successive della costruzione della chiesa.

In conclusione : con i dati verificati in questo documento si è cercato di dare il via alla ricerca alfine di tracciare un quadro generale del problema, visto che lo studio relativo all'inserzione di ceramiche acustiche nelle architetture mostra lacune notevoli per quanto riguarda i dati provenienti dagli scavi, tanto per il caso specifico albanese che in un contesto più generale. Ed è in questa prospettiva che si colloca il presente contributo, che chi scrive si augura possa apportare un ulteriore tassello al quadro già tracciato nell'ottimo libro di B. PALAzZo-BERTHOLON e J.-Ch. VAlière (dir.), Archéologie du son. Les dispositifs de pots acoustiques dans les édifices anciens (Paris, 2012) - che rappresenta lo sviluppo più recente in questo campo di studi, con esempi tratti da architetture medievali francesi, svizzere, tedesche, spagnole e croate.

Reçu : 18 septembre 2013 - Accepté : 6 décembre 2013 


\section{NOTES}

1. T. GјокA, «Gjoka, Mbi vlerat akustike të arkitekturës së traditës », Monumentet, 1 (1981), p. 45-50.

2. S. MUÇAJ, K. LAKO, E. HOBDARI e Y. VITALIOTIS, « Rezultatet e gërmimeve në bazilikën e Shën Janit, Delvinë (2001-2003) », Candavia, 1 (2004), p. 93-123. Nel 2005 gli scavi sono proseguiti alla scoperta della cripta sotto il pavimento della navata sud.

3. S. MUÇAJ et alii, « Rezultatet e gërmimeve... », ibid., p. 115-117.

4. Le anfore assomigliano ai tipi Otranto 1 e 2 e Paul Arthur mette l'accento sulla possibilità di una loro origine dalla regione della Puglia, sudest dell'Italia : P. ARTHUR, M.P. CAGGIA, G.P. CIONGOLI, V.V. MELISSANO, H. PATTERSON e P. ROBERTS, « Fornaci altomedievali ad Otranto. Nota preliminare ", Archeologia Medievale, 19 (1992), p. 91-122 ; P. ARTHUR e R. AURIEMMA, «A Search for Italian Wine. Middle Byzantine and Later Amphoras from Southern Puglia », INA Quarterly, XXIII/4 (1996), p. 14-17. Questo tipo di anfora è stato rinvenuto negli scavi degli ultimi anni a Teba (Grecia) e Butrint (Albania). Gli autori sostengono che si tratti di prodotti di provenienza corintica, databili nel X-XI sec. : P. ARMSTRONG, "Byzantine Thebes: Excavations on the Kadmeia, 1980 », The AnnualBritish School at Athens, 88 (1993), p. 301, fig. 4, 16 e 17 ; R. HODGES, W. BOWDEN, K. LAKO, Byzantine Butrint. Excavations and Surveys 1994-1999, Oxford, 2004. Allo stesso tempo sono molto diffuse nelle regioni dei Balcani e Bakirdzis gli mette nel quinto gruppo, con il corpo prolungato e manico che supera in altezza il livello dell'orlo, cronologicamente appartengono al periodo bizantino, databili attorno al X-XII sec.: Ch. BAKIRTZIS, Byzantine Tsoukalolagena. A contribution to the study of the names, shapes, and uses of the fireproof cooking pots, transport vessels and storage containers, Athens, 1989, p. 80.

5. P. ARTHUR, «Amphorae for bulk transport ", in F. D' ANDRIA e D. B. WHITEHOUSE (eds.), Excavations at Otranto II, Galatina, 1992, p. 108, fig. 6.6.477. Un esempio simile con la Tav. 6. 24, ma pitturato con fasce più larghe color rosso in marrone, è stato trovato negli scavi di Otranto, nel contesto della fase IV, databile grazie alle monete nel X sec.; E. BOLDRINI e F. GRASSI, « Alle radici dei castelli : indizi di economia curtense nei reperti ceramici della Toscana meridionale", in R. FIORILLO e P. PEDUTO (ed.), III Congresso Nazionale di Archeologia Medievale, Castello di Salerno, Complesso di Santa Sofia (Salerno, 2003), Firenze, 2003, p. 69, fig. 2. 21 (X sec.).

6. Un simile sinthronon con la platea di legno, lo troviamo nel periodo paleocristiano della città di Bylis (basilica A, D e la cattedrale).

7. Vedi supra, n. 1.

8. F. CANTINI, Archeologia urbana a Siena. L'area dell'Ospedale di Santa Maria della Scala prima dell'Ospedale. Altomedioevo, Firenze, 2005, p. 85, tav. 17, 4.89 (diam. $17 \mathrm{~cm}$; X-XII sec.).

9. F. CANTINI, Il castello di Montarrenti. Lo scavo archeologico 1982-1987. Per la storia della formazione del villaggio medievale in Toscana (secc. VII-XV), Firenze, 2003.

10. Il loro colore e' : Munsell Soil Color Charts. Revised washable edition 1998, 5Y 7/3 - 10YR 7/4.

11. F. CANTINI, Archeologia urbana..., op. cit., p. 127, fig. $I I .8 .8$ diametro $12 \mathrm{~cm}$, datato seconda metà VIII-IX sec.

12. P. ARTHUR, "Aspects of Byzantine economy : an evaluation of amphora evidence from Italy ", in V. DÉROCHE e J.-M. SPIESER (dir.), Recherches sur la céramique Byzantine, 
Athens, 1989 (supplément au Bulletin de correspondance Hellénique, 18), p. 88-89, fig. 10 (Italian Byzantine).

13. J. VRoom, Byzantine to Modern Pottery in the Aegean: An Introduction and Field Guide, Utrecht, 2005, p. 95, fig. 13.3, tipo databile attorno X-XI sec.

14. Il loro utilizzo e' accertato in Svizzera: V. Desarnaulds, Y. Loerincik e A.P.O. Carvalho, «Efficiency of 13th century acoustic ceramic pots in two Swiss churches ", letto a Noise-Con 2001, Portland, Maine, 29-31 October 2001; Grecia : F.A. Cuteri e E. Di Fede, «Bacini e vasi acustici nelle chiese del territorio di Megara (Attica-Grecia) », IX Congresso Internazionale AIECM2, Venezia, Dorsoduro, Auditorium Santa Margherita - Ca' Dolfin, 2009, in stampa. In Italia la chiesa di Panaghia di Rossano Calabro del X-XI secolo [San Bartolomeo del Fossato a Sampierdarena al XII secolo: F.A. Cuteri, « Vasi acustici nelle chiese bizantine della Calabria », in G. Volpe e P. Favia (dir.), V Congresso Nazionale di Archeologia Medievale, Foggia-Manfredonia, 2009, Firenze, 2009, p. 757-760], è contemporanea della chiesa di S. Giovanni a Delvinë ; in Croazia li troviamo nella Dalmazia del sud, dove nel Medioevo venne sviluppato un particolare modello architettonico di chiesa con una cupola [T. Marasović, "Patere i amfore u svodovima ranosrednjovjekovnih crkava u Dalmaciji ", Starohrvatska prosvjeta, iii serija, 30 (2003), p. 113-125]. Più di 200 chiese in tutta l'Europa hanno utilizzato vasi per migliorare l'acustica, la metà delle quali si trovano in Francia: B. Palazzo-Bertholon e J.-Ch. Valière, «Les vases acoustiques dans les églises médiévales et modernes: un programme d'étude interdisciplinaire ", Colloque Medieval Europe, Paris, 2007, 16 p., [http://medieval-europe-paris-2007.univ-paris1.fr/ B.\%20Palazzo-Bertholon\%20et\%20al..pdf]; P. Carvalho, J.-Ch. Valière e B. PalazzoBertholon, «Les vases acoustiques dans les églises médiévales : analyse des sources et études de cas ", in Y. Delomier (dir.), Techniques de construction dans les églises et les monastères, Saint-Germain-Laval, 2010, p. 19-43.

15. Il caso di studio della chiesa di S. Kolli (S. Nicola) e' pubblicato in T. GJOKA, «Gjoka, Mbi vlerat... », op. cit. La chiesa e del tipo a cupola sono databili attorno al XI-XII sec. [A. MEKSI, Arkitektura e kishave të Shqipërisë (shekujt VII - XV), Uegen, Tirana, 2004, fig. 80].

16. J. W. HAYES, Excavations at Saraçhane in Istanbul II. The pottery, Princeton, 1992, p. 35 (X sec./X-XI sec.) ; D. PAPANIKOLA-BAKIRTZIS, «Medieval Pottery from Enkomi, Famagusta ", in V. DÉROCHE e J.-M. SPIESER (dir.), Recherches sur la céramique..., op. cit., p. 49, fig. 6, 7 (X-XII sec.).

17. J. W. HAYES, Excavations at Saraçhane..., op. cit., p. 35.

18. K. DARK, Byzantine pottery, Stroud, 2001, p. 124, fig. 25, 26 e 27 (IX-XI sec.).

19. J. W. HAYES, Excavations at Saraçhane..., op. cit., p. 35 ; D. PAPANIKOLA-BAKIRTZIS, « Medieval Pottery... », op. cit., p. 51, fig. 8, a (X-XII sec.).

20. A. LANE, Early Islamic pottery. Mesopotamia, Egypt and Persia, London, 1947, p. 12, fig. 7, b ; C. H. MORGAN, Corinth XI: The Byzantine Pottery, Cambridge Mass., 1942, p. 88 fig. 66 ( $n^{\circ}$ 605), fig. 73 ( $\left.n^{\circ} 739\right)$ (XI sec.); T. TREPTOW e D. WHITCOMB, Daily life ornamented. The medieval Persian city of Rayy, Chicago, 2007, p. 34 (OIM A115003), IX-XI sec. La ceramica islamica si è diffusa nel sud del Mediterraneo durante il regno della dinastia Abaside, e fu adottato dagli artigiani locali specialmente nel sud dell'Italia e della Sicilia. Molto probabilmente i vasi usati come bacini nella chiesa di S. Giovanni a Delvinë sono stati importati da questi territori. 
21. C. H. MORGAN, Corinth XI..., ibid., p. 101, fig. 77 ( $\mathrm{n}^{\circ} 754$ ) (XI sec.) ; K. DARK, Byzantine pottery..., op. cit., p. 140-141, fig. 39 ; J. VROOM, Byzantine to Modern Pottery..., op. cit., p. 103, fig. 17.3.

INDEX

Mots-clés : céramique, vase acoustique

Index géographique : Albanie/Delvinë

\section{AUTEUR}

\section{SUELA XHYHERI}

Università di Tirana. Qëndra e studimeve albanologjike, Tiranë 